\title{
Upregulation of cholesterol 24-hydroxylase following hypoxia-ischemia in neonatal mouse brain
}

\author{
Fuxin $\mathrm{Lu}^{1}$, Jun $\mathrm{Zhu}^{2}$, Selena Guo ${ }^{3}$, Brandon $\mathrm{J} \mathrm{Wong}^{4}$, Farid F Chehab ${ }^{2}$, Donna M Ferriero ${ }^{1,5}$ and Xiangning Jiang ${ }^{1}$
}

BACKGROUND: Maintenance of cholesterol homeostasis is crucial for brain development. Brain cholesterol relies on de novo synthesis and is cleared primarily by conversion to 24S-hydroxycholesterol (24S-HC) with brain-specific cholesterol 24-hydroxylase (CYP46A1). We aimed to investigate the impact of hypoxia-ischemia $(\mathrm{HI})$ on brain cholesterol metabolism in the neonatal mice.

METHODS: Postnatal day 9 C57BL/6 pups were subjected to $\mathrm{HI}$ using the Vannucci model. CYP46A1 expression was assessed with western blotting and its cellular localization was determined using immunofluorescence staining. The amount of brain cholesterol, 24S-HC in the cortex and in the serum, was measured with enzyme-linked immunosorbent assay (ELISA).

RESULTS: There was a transient cholesterol loss at $6 \mathrm{~h}$ after $\mathrm{HI}$. CYP46A 1 was significantly upregulated at 6 and $24 \mathrm{~h}$ following $\mathrm{HI}$ with a concomitant increase of $24 \mathrm{~S}-\mathrm{HC}$ in the ipsilateral cortex and in the serum. The serum levels of $24 \mathrm{~S}-\mathrm{HC}$ correlated with those in the brain, as well as with necrotic and apoptotic cell death evaluated by the expression of spectrin breakdown products and cleaved caspase- 3 at 6 and $24 \mathrm{~h}$ after $\mathrm{HI}$.

CONCLUSION: Enhanced cholesterol turnover by activation of CYP46A1 represents disrupted brain cholesterol homeostasis early after neonatal HI. 24S-HC might be a novel blood biomarker for severity of hypoxic-ischemic encephalopathy with potential clinical application.

H uman brain cholesterol, which constitutes $25 \%$ of total body cholesterol, is crucial for brain development because of its importance in membrane integrity, myelination, synaptogenesis, and neurotransmission $(1,2)$. The majority of brain cholesterol is stored in myelin sheaths, and the rest in the membranes of neurons, glial cells, and other cellular elements. To accommodate rapid brain growth in the neonatal period, the rates of cholesterol biosynthesis and accretion are the highest during this stage (first 3 weeks after birth in the rodents), a critical time for neuroplasticity, and then decline with age to reach a constant cholesterol level at adulthood (3-6). As cholesterol carried in lipoproteins in the blood cannot cross the blood-brain barrier, brain cholesterol is produced exclusively from de novo synthesis $(3,7)$ using Acetyl-CoA as the starting material and HMG-CoA reductase (HMGCR) as the rate-limiting enzyme. However, 24Shydroxycholesterol (24S-HC), its metabolite that is generated through hydroxylation by cholesterol 24-hydroxylase (CYP46A1), is capable of traversing the blood-brain barrier and entering circulation to the liver for excretion $(4,7)$. CYP46A1 is brain-specific $(4,8)$; thus, most circulating $24 \mathrm{~S}$ HC has a cerebral origin (9) and the serum 24S-HC level could be an indication of brain cholesterol metabolism (10). In fact, plasma $24 \mathrm{~S}-\mathrm{HC}$ has been used as a surrogate marker of neuronal loss and brain atrophy in neurodegenerative diseases such as Alzheimer's disease, Parkinson's disease, and multiple sclerosis (11).

Cholesterol biosynthesis involves multiple enzymatic reactions and is an oxygen-consumptive process that requires 11 oxygen molecules for the conversion of Acetyl-CoA to cholesterol (12). Therefore, cholesterol synthesis is sensitive to reduced $\mathrm{O}_{2}$ availability and is limited in the conditions of HI. This is evidenced by two studies in neonatal HI rats showing chronic loss of brain cholesterol lasting at least 3 days or 3 months following the insults $(13,14)$. Unfortunately, there are no additional investigations on the changes of cholesterol metabolism in response to $\mathrm{HI}$ in the immature brain and the underlying mechanisms. Recent in vitro studies suggested that oxidative stress (6) and endogenous neurotransmitters upregulate CYP46A1, with glutamate eliciting the highest increase in CYP46A1 activity (15). These are wellaccepted mechanisms that are associated with brain damage in neonatal HI encephalopathy (HIE), the clinical syndrome of brain dysfunction in the newborns with few tools for diagnosis and intervention (16). Studies on regulation of cholesterol homeostasis in the developing brain, as well as its disturbance after $\mathrm{HI}$ at early postnatal stage, would allow for a better understanding of lipid disorders and their involvement in HIE gray and white matter injury.

The present study focused on the responses of cholesterol metabolism after neonatal HI on postnatal day 9 (P9) mice, an

\footnotetext{
${ }^{1}$ Department of Pediatrics, University of California San Francisco, San Francisco, California; ${ }^{2}$ Department of Laboratory Medicine, University of California San Francisco, San Francisco, California; ${ }^{3}$ Dougherty Valley High School, San Ramon, California; ${ }^{4}$ University of California Berkeley, Berkeley, California; ${ }^{5}$ Department of Neurology, University of California San Francisco, San Francisco, California. Correspondence: Xiangning Jiang (xiangning.jiang@ucsf.edu)

Received 13 November 2017; accepted 2 March 2018; advance online publication 2 May 2018. doi:10.1038/pr.2018.49
} 


\section{Cholesterol metabolism in neonatal brain}

age equivalent to full-term human infants. We hypothesized that the brain-specific cholesterol hydroxylase, CYP46A1, is activated following neonatal $\mathrm{HI}$ leading to an enhanced production of $24 \mathrm{~S}-\mathrm{HC}$ in the brain, and in the circulation. Herein, we demonstrated an increase and correlation of the 24S-HC levels in the serum and in the brain, and, importantly, a significant positive correlation between serum 24S-HC levels and cortical injury evaluated by the expression of spectrin breakdown products (SBDPs) and cleaved caspase3 , representing activation of necrosis and/or apoptosis, at 6 and $24 \mathrm{~h}$ after HI. These findings suggested a possibility of using $24 \mathrm{~S}-\mathrm{HC}$ as a potential blood biomarker for severity of HIE brain injury.

\section{METHODS}

All animal experiments were approved by the University of California San Francisco institutional animal care and use committee. C57BL/6 mice (Charles River Laboratory, Hollister, CA) with litters were allowed food and water ad libitum. Both sexes were used on P9.

\section{Neonatal Brain HI}

Neonatal HI was performed using the Vannucci model. On P9, the pups underwent left common carotid artery coagulation through a vertical midline neck incision under isoflurane anesthesia $(2-3 \%$ isoflurane, balanced oxygen) to induce unilateral ischemia. The animals were allowed to recover for $1 \mathrm{~h}$ with their dam and then exposed to $60 \mathrm{~min}$ of hypoxia in a humidified chamber at $37^{\circ} \mathrm{C}$ with $10 \%$ oxygen/balanced nitrogen to induce global hypoxia. Shamoperated control animals received isoflurane anesthesia and exposure of the left common carotid artery without coagulation and hypoxia. The animals were dissected immediately after hypoxia $(0 \mathrm{~h})$ or returned to their dams until they were killed at $1,6,24,48$, or $72 \mathrm{~h}$ after the procedure.

\section{Sample Preparation}

The cortices were dissected and quickly sliced into small pieces on ice, mixed, and divided into three equal fractions for protein (western blotting), cholesterol, or $24 \mathrm{~S}-\mathrm{HC}$ measurements, respectively. For protein purification, the tissue was homogenized in radioimmunoprecipitation assay buffer (R0278, MilliporeSigma, Temecula, CA) with protease and phosphatase inhibitors, followed by centrifugation at 14,000 r.p.m. for $15 \mathrm{~min}$. The supernatant was saved and the protein concentration was measured with BCA kit (Pierce Biotechnology, Rockford, IL). For 24S-HC assay, the tissue was sonicated with $95 \%$ ethanol; after centrifugation at $7,000 \mathrm{~g}$ for $5 \mathrm{~min}$, the supernatant (A) was collected and the pellet was sonicated again in the extract buffer (ethanol:dichloromethane $(1: 1 ; \mathrm{v} / \mathrm{v})$ ) followed by centrifugation at $7,000 \mathrm{~g}$ for $5 \mathrm{~min}$. The resultant supernatant (B) was combined with supernatant $A$ and dried with speed vacuum. The dried pellet was rehydrated by adding $8 \mu \mathrm{l}$ of $95 \%$ ethanol and $242 \mu \mathrm{l}$ of assay buffer 40 (24S-hydroxycholesterol ELISA (enzyme-linked immunosorbent assay) Kit, ab204530, Abcam, Cambridge, MA). For cholesterol measurement, the tissue was extracted with $200 \mu \mathrm{l}$ of chloroform:isopropanol:IGEPAL CA-630 (7:11:0.1) in a microhomogenizer and spun at $13,000 \mathrm{~g}$ for $10 \mathrm{~min}$ to remove insoluble material. The organic phase was air-dried at $50{ }^{\circ} \mathrm{C}$ for $15 \mathrm{~min}$ to remove chloroform. The samples were then dried with speed vacuum and dissolved in $200 \mu \mathrm{l}$ cholesterol assay buffer (Cholesterol Quantitation Kit, MAK043, MilliporeSigma) for cholesterol quantification.

Serum was collected from the same animals using a BD SST microtainer capillary blood collection tube with serum separator (\#365967, BD, Franklin Lakes, NJ).

\section{Western Blotting}

An equal amount of protein samples $(40 \mu \mathrm{g})$ was applied to $4-12 \%$ Bis-Tris SDS polyacrylamide gel electrophoresis and transferred to polyvinylidene difluoride membrane at $30 \mathrm{~V}$ overnight at $4{ }^{\circ} \mathrm{C}$. After blocking with TBS containing $0.05 \%$ Tween 20 (TBST) buffer with $5 \%$ milk for $1 \mathrm{~h}$, the blots were probed overnight at $4{ }^{\circ} \mathrm{C}$ with the following primary antibodies: anti-cholesterol-24-hydroxylase 1A7 (1:500; MAB2259, MilliporeSigma), anti- $\alpha$-spectrin $(1: 5,000$, MAB1622, MilliporeSigma), anti-cleaved caspase-3 (1:1,000, \#9664, Cell Signaling Technology, Danvers, MA), and anti- $\beta$-actin $(1: 5,000$, sc-47778, Santa Cruz Biotechnology, Santa Cruz, CA). Appropriate secondary horseradish peroxidase-conjugated antibodies (Santa Cruz Biotechnology) were used. The protein signal was visualized with enhanced chemiluminescence and developed with a radiographic film. Image J software was used to measure the mean optical densities (OD) and areas of protein signal on the film after scanning.

\section{Enzyme-Linked Immunosorbent Assay for 24S-HC Levels in the Cortex and Serum}

A competitive ELISA kit (ab204530, Abcam) was used to measure the levels of $24 \mathrm{~S}-\mathrm{HC}$ in the cortex and serum. A fresh set of $24 \mathrm{~S}-\mathrm{HC}$ standard was prepared at a range of $0.39-100 \mathrm{ng} / \mathrm{ml}$ and a clear 96 well plate coated with goat anti-rabbit IgG was used. Briefly, $100 \mu \mathrm{l}$ of standards and samples were pipetted into the appropriate wells. An aliquot of $50 \mu \mathrm{l}$ of the diluted biotin-labeled 24S-HC conjugate was then added to each well except for the blanks. Fifty microliters of the rabbit $24 \mathrm{~S}-\mathrm{HC}$ antibody that can bind to either $24 \mathrm{~S}-\mathrm{HC}$ in the samples or to the labeled $24 \mathrm{~S}-\mathrm{HC}$ conjugate was added to each well, except for the blanks and the nonspecific binding (NSB) wells. The plate was incubated for $1 \mathrm{~h}$ at room temperature (RT). After washing four times with $400 \mu \mathrm{l}$ of wash buffer, $200 \mu \mathrm{l}$ of streptavidinhorseradish peroxidase solution was pipetted into each well to bind to biotin-labeled 24S-HC conjugate. The plate was incubated for $30 \mathrm{~min}$ at RT. After the second wash, $200 \mu \mathrm{l}$ of $3,3^{\prime}, 5,5^{\prime}$-Tetramethylbenzidine substrate solution was added and incubated at RT for $30 \mathrm{~min}$. To stop the reaction, $50 \mu \mathrm{l}$ of stop solution $(1 \mathrm{~N} \mathrm{HCl})$ was added and the absorbance (OD) was read at $450 \mathrm{~nm}$ with a microplate reader (BioTek Synergy, Winooski, VT). The intensity of signal was inversely proportional to the levels of $24 \mathrm{~S}-\mathrm{HC}$ in the samples. For each standard and sample, the net OD = OD - NSB OD. A standard curve was made for the calculation of the levels of $24 \mathrm{~S}$ HC. The concentration of cortical 24S-HC was normalized to the wet weight of the starting tissue (ng/mg wet weight). The concentration of serum $24 \mathrm{~S}-\mathrm{HC}$ was normalized to the serum protein concentration (ng/mg protein).

\section{Cholesterol Measurement}

A cholesterol quantitation kit (MAK043, MilliporeSigma) was used to measure the concentration of cholesterol in the cortex. The samples prepared above were diluted at 1:10 with assay buffer and seven cholesterol standards $(0.02-0.14 \mu \mathrm{g} / \mu \mathrm{l})$ were prepared. Hundred microliters of standards and samples were pipetted into the appropriate wells of a clear 96-well plate. Fifty microliters of reaction mix buffer including cholesterol probe, cholesterol enzyme mix, and cholesterol esterase were added to each well. The plate was incubated at $37^{\circ} \mathrm{C}$ for $60 \mathrm{~min}$ and protected from light. The absorbance was read at $570 \mathrm{~nm}$ and the concentration of cholesterol in the samples was calculated using the standard curve. The levels of cortical cholesterol were normalized to the wet weight of the starting tissue ( $\mu \mathrm{g} / \mathrm{mg}$ wet weight).

\section{Immunofluorescent Staining}

At $24 \mathrm{~h}$ after $\mathrm{HI}$, the sham and injured mice were perfuse-fixed with $4 \%$ paraformaldehyde in $0.1 \mathrm{~mol} / 1$ phosphate buffer ( $\mathrm{pH} 7.4$ ). Brains were dissected and post-fixed in $4 \%$ paraformaldehyde overnight at $4{ }^{\circ} \mathrm{C}$, and then transferred to $30 \%$ sucrose in $0.1 \mathrm{~mol} / \mathrm{l}$ phosphate buffer for cryoprotection for 3 days. The brains were embedded in optimal cutting temperature (O.C.T.) compound and cryosections were cut at $16 \mu \mathrm{m}$. The sections were defrosted and air-dried at RT 


\section{Articles | Lu et al.}

a

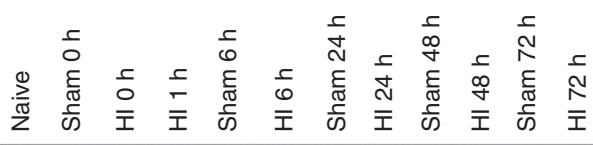

CYP46A

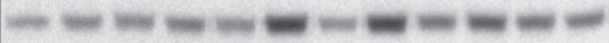

$\beta$-actin
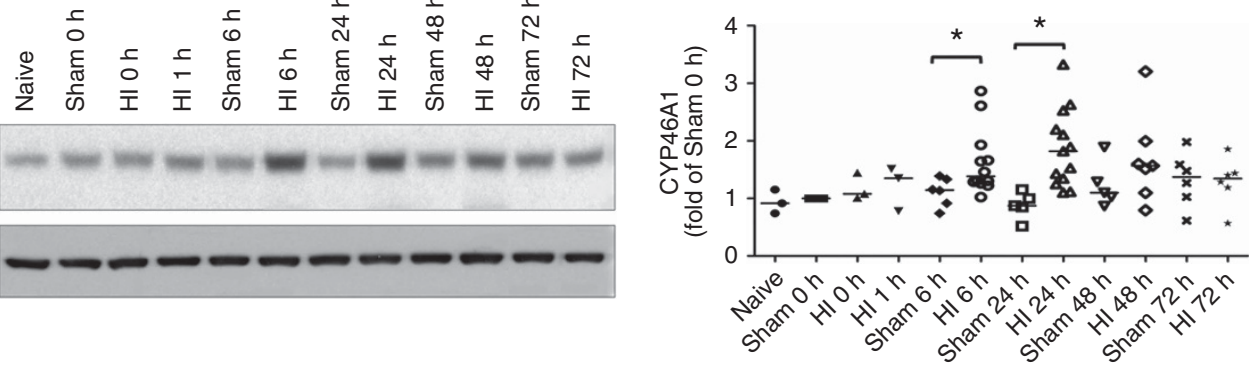

b
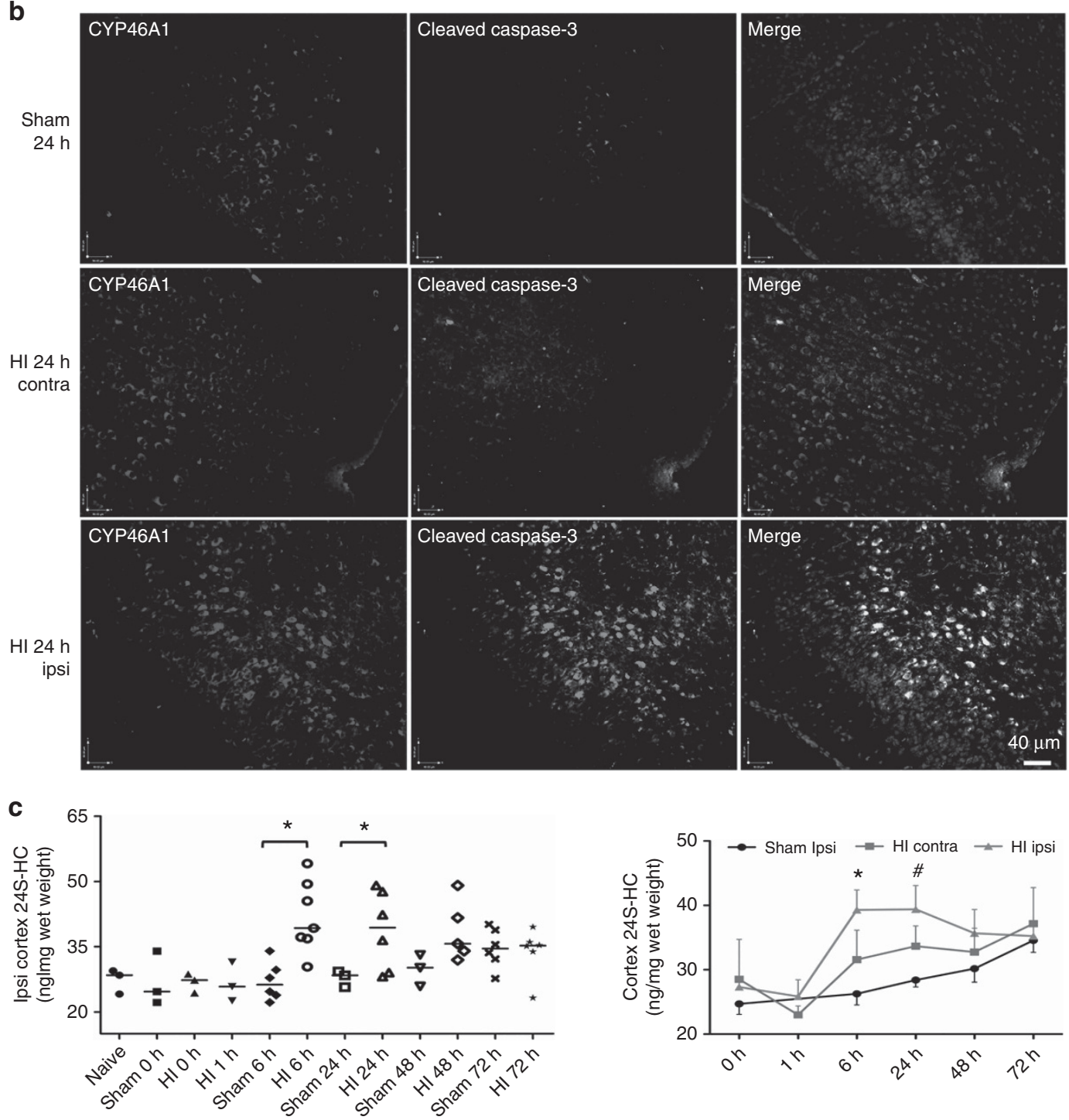

Figure 1. Upregulation of CYP46A1 with a concomitant increase of $24 \mathrm{~S}-\mathrm{HC}$ in the ipsilateral (ipsi) cortex after neonatal HI. (a) Protein expression of CYP46A1 was measured by western blotting at the indicated time points and was presented as the OD ratio to $\beta$-actin and normalized to the values of sham $0 \mathrm{~h}$ (graph on the right, sham vs. HI: ${ }^{*} P=0.0339$ at $6 \mathrm{~h}$; ${ }^{*} P=0.0026$ at $24 \mathrm{~h} ; n=5-6$ for sham animals, $n=6-12$ for $\mathrm{HI}$ animals at $6-72 \mathrm{~h}$ ). (b) Immunofluorescent staining with anti-CYP46A1 antibody at $24 \mathrm{~h}$ after $\mathrm{HI}$ showed enhanced expression in the ipsi cortex than that in the contralateral side and in the sham animals (left panels). CYP46A1 staining in the ipsi cortex was largely colocalized with the expression of cleaved caspase-3 (middle panels). (c) Increased production of $24 \mathrm{~S}-\mathrm{HC}$ ( $\mathrm{ng} / \mathrm{mg}$ tissue wet weight) in the ipsi cortex at 6 and $24 \mathrm{~h}$ after $\mathrm{HI}$ (sham vs. HI: ${ }^{*} P=0.0167$ at $6 \mathrm{~h}$; ${ }^{*}=0.0085$ at $24 \mathrm{~h} ; n=3-6$ for sham animals, $n=5-7$ for HI animals at 6-72 h). The time course of the changes in $24 \mathrm{~S}-\mathrm{HC}$ in the sham-, contra-, and ipsi cortex is shown on the right. At $6 \mathrm{~h}, 24 \mathrm{~S}-\mathrm{HC}$ in the ipsi cortex was higher than that in the contralateral side (ipsi- vs. contra-: ${ }^{*} P=0.0493$ ). "Significant difference between the $\mathrm{HI}$ ipsi- and the sham animals only, but not between the HI ipsi- and HI contralateral hemisphere. 24S-HC, 24Shydroxycholesterol; HI, hypoxia-ischemia; OD, optical density. 
for $2 \mathrm{~h}$. After washing with phosphate-buffered saline (PBS), sections were boiled in $0.01 \mathrm{M}$ sodium citrate buffer ( $\mathrm{pH}$ 6.0, with $0.05 \%$ Tween 20) at $90{ }^{\circ} \mathrm{C}$ for $30 \mathrm{~min}$ to retrieve antigen. After washing with PBS, the sections were blocked with blocking buffer (10\% goat serum and $0.1 \%$ Triton X-100 in PBS) for $1 \mathrm{~h}$ and then incubated overnight at $4{ }^{\circ} \mathrm{C}$ with mouse anti-CYP46A1 antibody (1:50, MAB2259, MilliporeSigma), paired with another rabbit antibody that labels the following different cell types: anti-NeuN for neurons (1:500, \#ab177487, Abcam), anti-glial fibrillary acidic protein (GFAP) for astrocytes (1:500, \#Z0334, Dako, Santa Clara, CA), anti-Iba1 for microglia (1:1,000, \#019-19741, Wako Chemicals, Richmond, VA), or anti-myelin basic protein (MBP) for oligodendrocytes (1:200, \#78896, Cell Signaling Technology). Antibody against cleaved caspase-3 (1:500, \#9664, Cell Signaling Technology) was used for double staining with anti-CYP46A1 to study their colocalization. After washing with $0.025 \%$ Tween/PBS for three times, the sections were incubated with goat anti-mouse Alexa Fluor 568 (1:500; \#A-11004, Invitrogen, Grand Island, NY) and goat anti-rabbit Alexa Fluor 488 (1:500, \#A-11034, Invitrogen) for $1 \mathrm{~h}$ at RT and exposed to 4',6-diamidino-2-phenylindole (DAPI) for $5 \mathrm{~min}$ and then coverslipped with ProLong Diamond antifade reagent (Invitrogen). Images were taken with Leica TCS SP5 Spectral Confocal Microscope.

\section{Statistical Analysis}

SAS Wilcoxon-Mann-Whitney test was used to evaluate the OD values of protein expression in western blotting, brain concentrations of cholesterol, and the cortical and serum levels of 24S-HC. Pearson's correlation coefficient analysis was used to evaluate the correlation between cortical and serum levels of $24 \mathrm{~S}-\mathrm{HC}$, and between serum $24 \mathrm{~S}-\mathrm{HC}$ and protein levels of SBDP at $145 / 150 \mathrm{kDa}$, SBDP at $120 \mathrm{kDa}$, or cleaved caspase-3. Differences were considered significant at $P<0.05$.

\section{RESULTS}

\section{Upregulation of CYP46A1 with a Concomitant Increase of 24S-} $\mathrm{HC}$ in the Brain Following Neonatal $\mathrm{HI}$

To investigate how cholesterol metabolism is influenced by neonatal HI, we measured the protein expression of CYP46A1, the enzyme responsible for elimination of excess cholesterol from the brain, at various post-HI time points for up to 3 days. As shown in Figure 1a, CYP46A1 was significantly upregulated at 6 and $24 \mathrm{~h}$ after $\mathrm{HI}$ compared to the sham animals at the same time points, and declined at 48 and $72 \mathrm{~h}$ (sham vs. HI: $P=0.0339$ at $6 \mathrm{~h} ; P=0.0026$ at $24 \mathrm{~h}$; $n=5-6$ for sham animals, $n=6-12$ for HI animals at $6-72 \mathrm{~h}$ ). The increased expression of CYP46A1 was confirmed by immunofluorescent staining with anti-CYP46A1 antibody at $24 \mathrm{~h}$ after HI, when the fluorescent intensity was much higher in the ipsilateral cortex compared with that of the sham animals and the contralateral hemisphere (left panels in Figure 1b). It is interesting that the expression of CYP46A1 in the ipsilateral cortex was largely localized to the cells undergoing apoptotic cell death as visualized by the expression of cleaved caspase-3 (Figure 1b).

The pattern of $24 \mathrm{~S}-\mathrm{HC}$ levels in the ipsilateral cortex was similar to that of CYP46A1 expression with a marked increase at 6 and $24 \mathrm{~h}$ post-HI compared with that of the sham controls (Figure 1c, sham vs. HI: $P=0.0167$ at $6 \mathrm{~h} ; P=0.0085$ at $24 \mathrm{~h} ; n=3-6$ for sham animals, $n=5-7$ for HI animals at $6-72 \mathrm{~h}$ ). At $6 \mathrm{~h}, 24 \mathrm{~S}-\mathrm{HC}$ in the ipsilateral cortex was higher than that in the contralateral side, too (Figure 1c, HI ipsilateral vs. HI contralateral: $P=0.0493$ at 6 h). HI-induced overproduction of $24 \mathrm{~S}-\mathrm{HC}$ in the ipsilateral cortex sustained at $48 \mathrm{~h}$, although the difference was not statistically significant compared with that in the sham animals (Figure 1c, sham vs. HI: $P=0.0526$ at $48 \mathrm{~h}$ ). There was a trend of more $24 \mathrm{~S}-\mathrm{HC}$ produced in the contralateral cortex (underwent hypoxia) at 6 and $24 \mathrm{~h}$; however, the differences were not significant (Figure 1c, right panel). These results demonstrated that the upregulated CYP46A1 was functional, which led to robust cholesterol turnover early after $\mathrm{HI}$, along with an enhanced production of its metabolic product $24 \mathrm{~S}-\mathrm{HC}$ in the injured cortex, but not in the non-injured contralateral hemisphere.

To determine whether the increased cholesterol metabolism following neonatal $\mathrm{HI}$ would diminish brain cholesterol amount, we measured cholesterol levels in the sham and

a

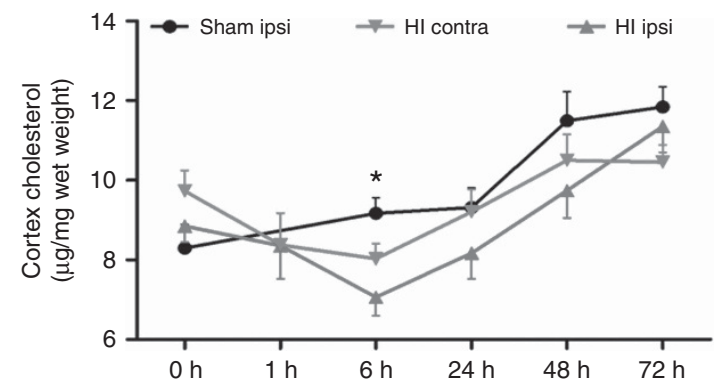

b

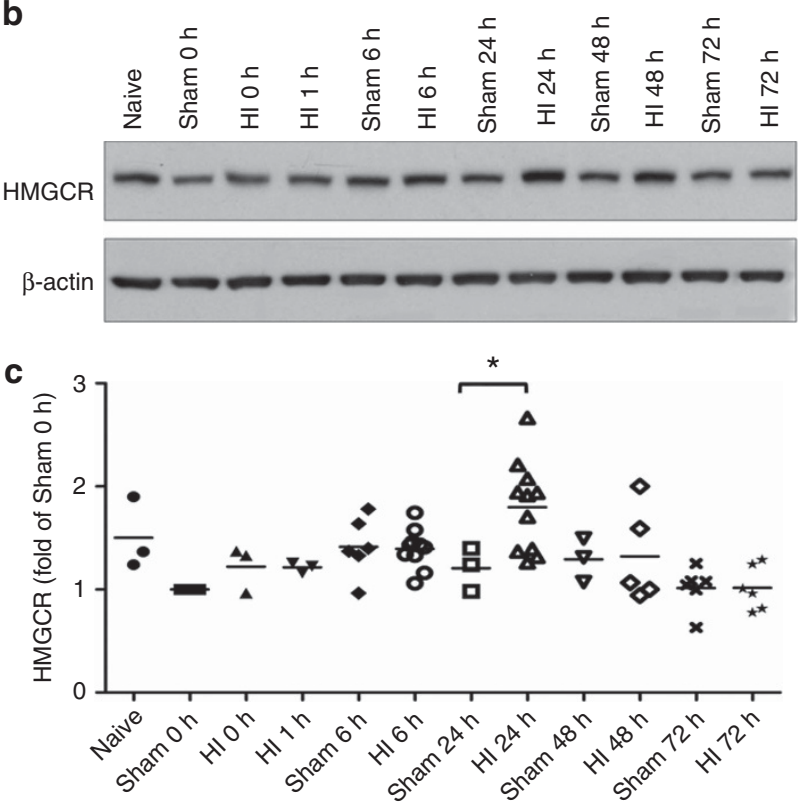

Figure 2. Transient cholesterol loss in the ipsilateral cortex at $6 \mathrm{~h}$ after neonatal HI. (a) The time course of the changes in cholesterol amount in the sham-, contra-, and ipsilateral cortex following neonatal HI (sham vs. HI: ${ }^{*} P=0.0304$ at $6 \mathrm{~h} ; n=4-7$ for each time point). (b) Protein expression of HMGCR in the sham and HI-injured cortices was measured by western blotting at the indicated time points. (c) HMGCR expression is presented as the OD ratio to $\beta$-actin and is normalized to the values of sham $0 \mathrm{~h}$ (sham vs. HI: ${ }^{*} P=0.047$ at $24 \mathrm{~h}, n=3-11$ ). $24 \mathrm{~S}-\mathrm{HC}, 24 \mathrm{~S}$ hydroxycholesterol; HI, hypoxia-ischemia; HMGCR, HMG-CoA reductase; OD, optical density. 


\section{Articles | Lu et al.}

HI-injured ipsi- and contralateral cortex at the same time points. We found a significant, but transient loss of cholesterol at $6 \mathrm{~h}$ after $\mathrm{HI}$ in the ipsilateral cortex
(Figure 2a, sham vs. HI ipsi: $P=0.0304$ at $6 \mathrm{~h} ; n=7$ ), but not in the contralateral side (sham vs. HI contra: $P=0.1736$; $n=7$ ) compared with the sham animals. These changes

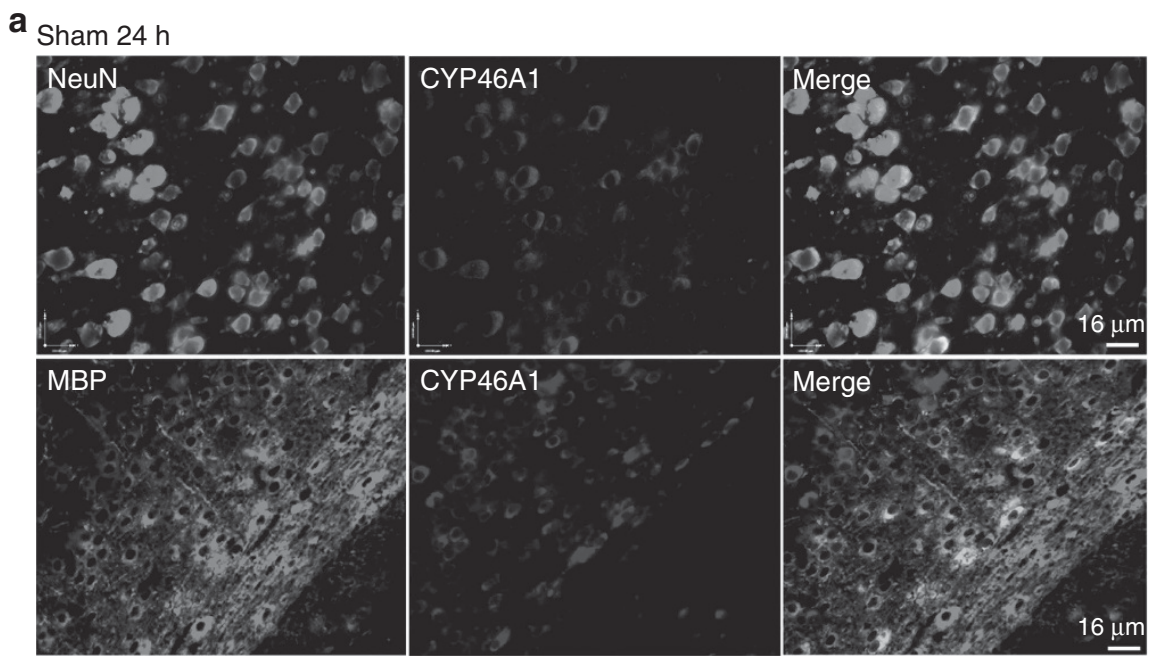

b $\mathrm{HI} 24 \mathrm{~h}$

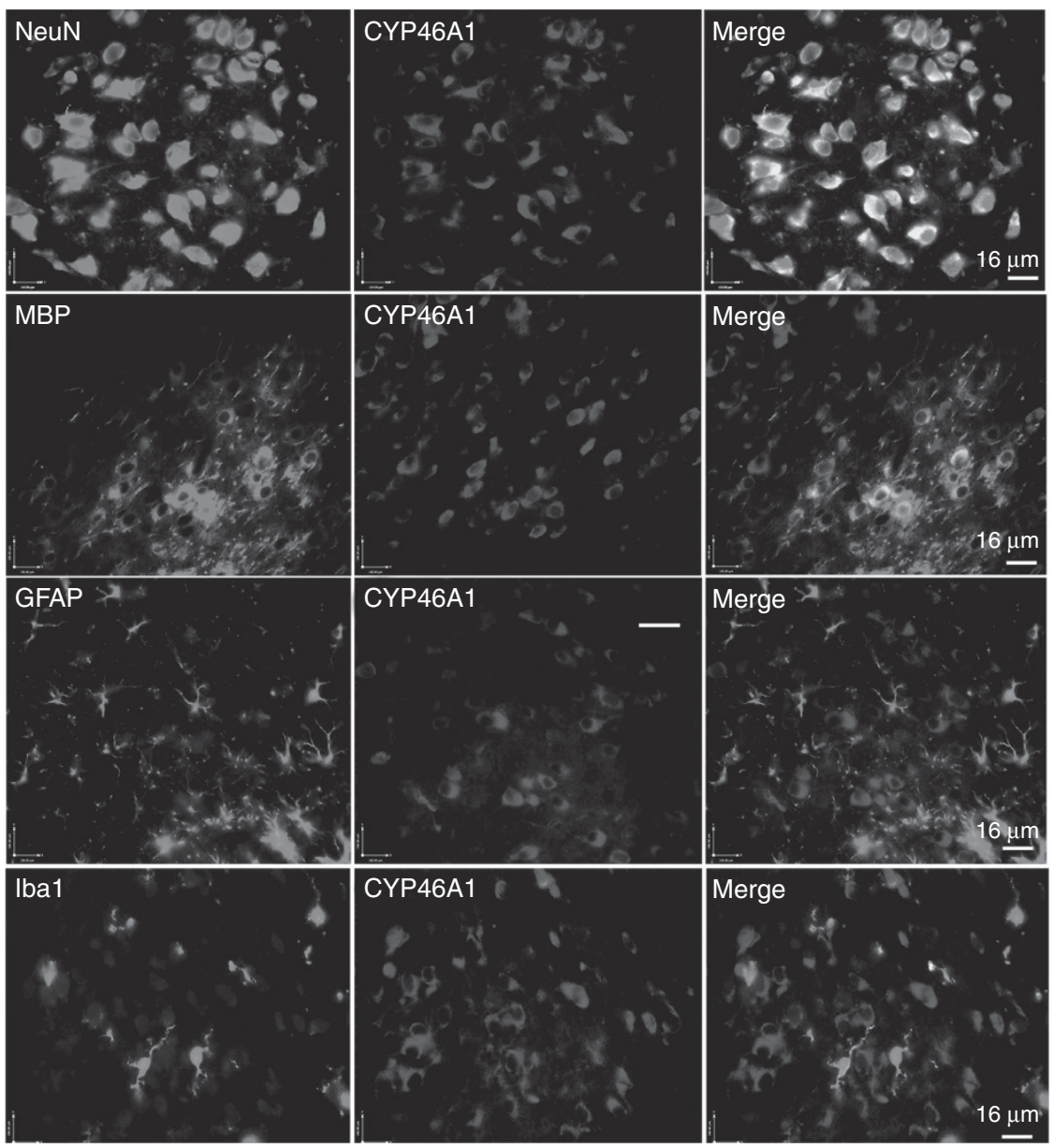

Figure 3. Expression of CYP46A1 in the neurons and oligodendrocytes. Images of double immunofluorescent staining with CYP46A1 antibody (red, middle panels) paired with another antibody specific for neuron (NeuN), oligodendrocyte (MBP), astrocyte (GFAP), or microglia (Iba1), shown in green on the left panels. The representative images from the sham (a) and HI-injured animals at $24 \mathrm{~h}$ after HI (b). 24S-HC, 24S-hydroxycholesterol; $\mathrm{HI}$, hypoxia-ischemia;. 


\section{Cholesterol metabolism in neonatal brain}

Articles

represented the net results of de novo cholesterol synthesis and turnover. The enhanced cholesterol hydroxylation/ breakdown may, at least in part, contribute to the cholesterol loss at $6 \mathrm{~h}$, whereas the elevated expression of HMGCR, the rate-limiting enzyme for cholesterol biosynthesis, at $24 \mathrm{~h}$ (Figure $2 \mathbf{b}, \mathbf{c}$, sham vs. HI: $P=0.047$ at $24 \mathrm{~h}$, $n=4-11)$ indicates feedback mechanisms for replenishment and re-establishment of cholesterol balance at $24 \mathrm{~h}$ and the time points thereafter.

\section{Expression of CYP46A1 in Neurons and Oligodendrocytes}

CYP46A1 was originally reported to be neuron-specific; however, several recent studies demonstrated its presence in glial cells, including microglia and astrocytes in different injury paradigms $(17,18)$. To study the cellular localization of CYP46A1 in our neonatal HI model, we stained the brain sections from the sham and injured pups (at $24 \mathrm{~h}$ post-HI) with CYP46A1 antibody paired with another antibody specific for neuron (NeuN), astrocyte (GFAP), oligodendrocyte (MBP), or microglia (Iba1). Figure 3 indicates that the enzyme was expressed in neurons (shown in the cortical region) and oligodendrocytes (shown in the corpus callosum) in both sham (Figure 3a) and the injured animals (Figure 3b). Colocalization was not found in the astrocytes and microglia in the sham (not shown) and injured brains (Figure 3b). The CYP46A1 staining was in the cytoplasm.

\section{Correlation of the 24S-HC Levels in the Serum and in the Brain after Neonatal HI}

As CYP46A1 converts brain cholesterol to the more soluble 24S-HC, which readily crosses blood-brain barrier, and this enzymatically generated oxysterol is specifically made in the brain, we asked whether there is an efflux of $24 \mathrm{~S}$-HC from the brain into the periphery circulation after HI. We measured the serum levels of 24S-HC and evaluated its relationship with brain levels. In line with the increased production in the brain, there was a boost of serum 24S-HC levels at $6 \mathrm{~h}$ after HI, which sustained for $48 \mathrm{~h}$ before returning to the sham values at $72 \mathrm{~h}$ (Figure $4 \mathrm{a}$, sham vs. HI, $P=0.0102$ at $6 \mathrm{~h} ; P=0.0046$ at $24 \mathrm{~h} ; P=0.0167$ at $48 \mathrm{~h} ; n=3-6$ for sham animals, $n=5-13$ for $\mathrm{HI}$ animals at $6-72 \mathrm{~h}$ ). There was a strong correlation of 24S-HC levels in the serum and in the ipsilateral cortex (Figure $4 \mathbf{b}, R^{2}=0.521, P<0.0001, n=40$ ), implicating the brain as the major source of circulating 24S-HC (note that the concentrations of $24 \mathrm{~S}-\mathrm{HC}$ in the serum were normalized to serum protein concentrations as $\mathrm{ng} / \mathrm{mg}$ protein, whereas in the brain they were normalized to the tissue wet weight as $\mathrm{ng} / \mathrm{mg}$ wet weight).

\section{Correlation of Serum 24S-HC Levels with Brain Injury at 6 and $24 \mathrm{~h}$ After HI}

As cholesterol is essential for membrane and myelin integrity, and CYP46A1 upregulation may cause membrane disruption or myelin breakdown leading to cell death and brain injury after HI, we hypothesized that increased serum 24S-HC values could forecast $\mathrm{HI}$-induced cell loss and gray/white
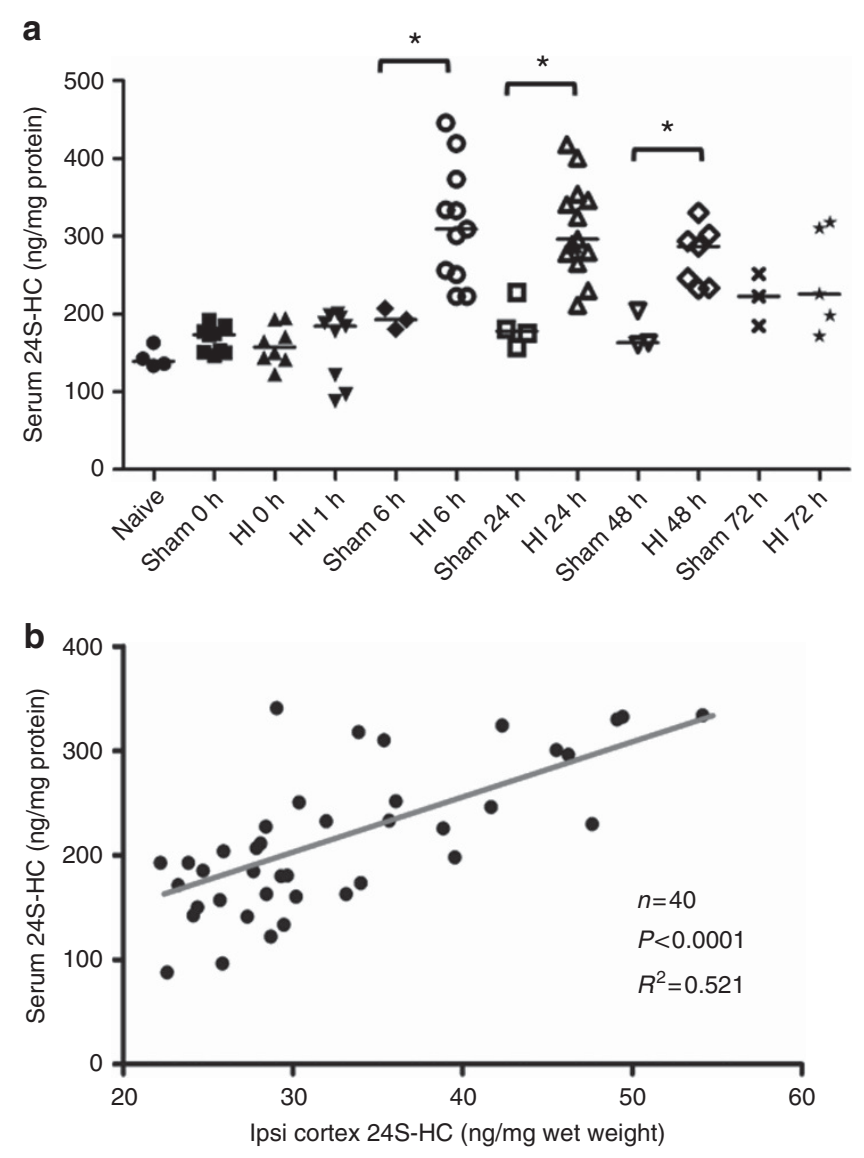

Figure 4. Correlation of the $24 \mathrm{~S}-\mathrm{HC}$ levels in the serum and in the ipsilateral cortex after neonatal HI. (a) Quantification of the serum levels of $24 \mathrm{~S}-\mathrm{HC}$ (ng/mg serum protein) at the indicated post-HI time points. Sham vs. $\mathrm{HI},{ }^{*} P=0.0102$ at $6 \mathrm{~h},{ }^{*} P=0.0046$ at $24 \mathrm{~h},{ }^{*} P=0.0167$ at $48 \mathrm{~h}$; $n=3-6$ for sham animals, $n=5-13$ for $\mathrm{HI}$ animals at $6-72 \mathrm{~h}$. (b) Correlation of $24 \mathrm{~S}-\mathrm{HC}$ levels in the serum and in the ipsilateral cortex. The samples used in Pearson's correlation coefficient analysis were those included in Figure 1c $\left(n=40, R^{2}=0.521, P<0.0001\right) .24 \mathrm{~S}-\mathrm{HC}, 24 \mathrm{~S}-$ hydroxycholesterol; HI, hypoxia-ischemia;.

matter damage. We assessed acute brain injury within 3 days with the protein expression of SBDP at $145 / 150$ and $120 \mathrm{kD}$ representing activation of necrosis and/or apoptosis, as well as the cleaved caspase- 3 , indicating apoptotic cell death. The expression of both SBDP at 145/150 and $120 \mathrm{kD}$ considerably increased at 6 and $24 \mathrm{~h}$ after $\mathrm{HI}$ compared with the sham animals (Figure 5a, for SBDP $145 / 150 \mathrm{kD}$, sham vs. HI, $P=0.0094$ at $6 \mathrm{~h}, P=0.0013$ at $24 \mathrm{~h}$. For SBDP $120 \mathrm{kD}$, sham vs. HI, $P=0.036$ at $6 \mathrm{~h}, P=0.0087$ at $24 \mathrm{~h}, n=4-6$ for sham animals, $n=6-15$ for $\mathrm{HI}$ animals from 6 to $72 \mathrm{~h}$ ). The increase in cleaved caspase- 3 levels lasted longer for $48 \mathrm{~h}$ and subsided at $72 \mathrm{~h}$ (Figure 6a, sham vs. HI, $P=0.0112$ at $6 \mathrm{~h}$; $P=0.0027$ at $24 \mathrm{~h} ; P=0.0228$ at $72 \mathrm{~h}, n=5-6$ for sham animals, $n=7-10$ for $\mathrm{HI}$ animals from 6 to $72 \mathrm{~h}$ ). There was a positive correlation between serum 24S-HC levels and SBDP $145 / 150 \mathrm{kD}$ at 6 and $24 \mathrm{~h}$ post-HI (Figure 5b, at $6 \mathrm{~h}$ : $R^{2}=0.713, P=0.002, n=10 ;$ at $24 \mathrm{~h}: R^{2}=0.656, P=0.003$, 
a

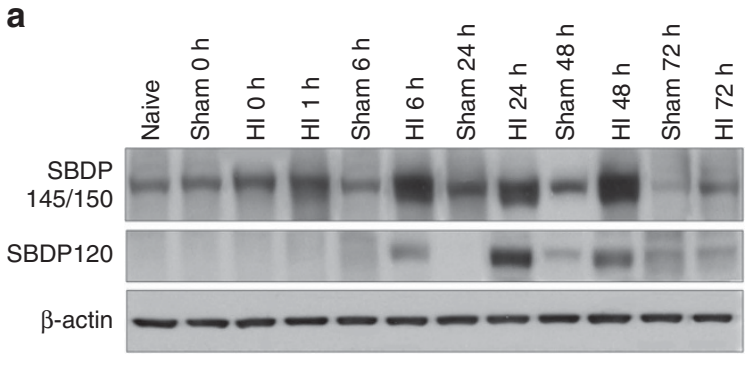

b
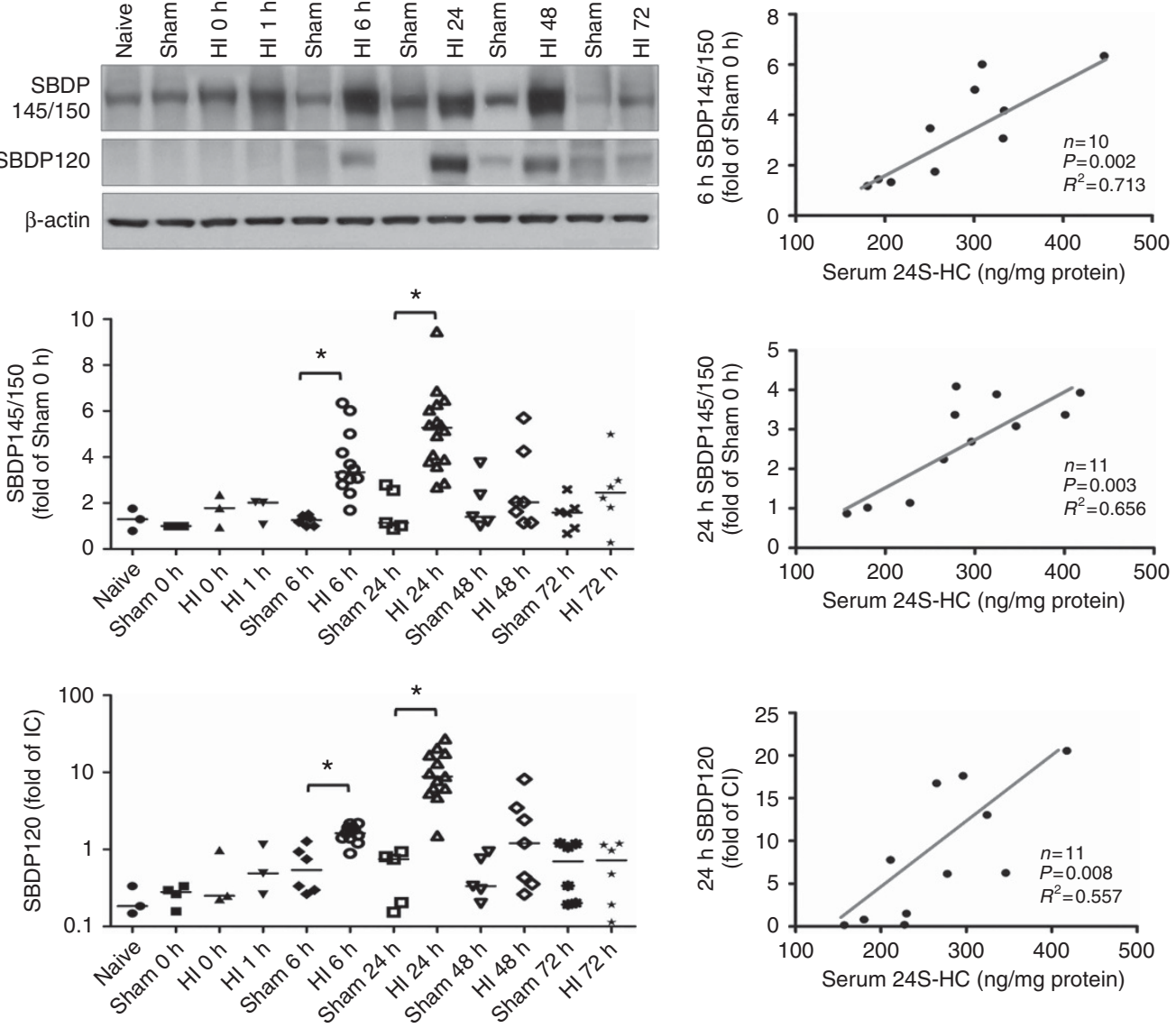

Figure 5. Correlation of the serum levels of 24S-HC with the expression of SBDPs at 6 and $24 \mathrm{~h}$ after neonatal HI. (a) Protein expression of SBDP145/150 kD and SBDP $120 \mathrm{kD}$ in the cortices was measured by western blotting at the indicated time points and was presented as the OD ratio to $\beta$-actin and normalized to the values of sham $0 \mathrm{~h}$ (for SBDP 145/150 kD), or an internal control (IC, for SBDP $120 \mathrm{kD}$ ). For SBDP $145 / 150 \mathrm{kD}$, sham vs. $\mathrm{HI},{ }^{*} P=0.0094$ at $6 \mathrm{~h},{ }^{*} P=0.0013$ at $24 \mathrm{~h}$. For SBDP $120 \mathrm{kD}$, sham vs. $\mathrm{HI},{ }^{*} P=0.036$ at $6 \mathrm{~h},{ }^{*} P=0.0087$ at $24 \mathrm{~h}(n=4-6$ for sham animals, $n=6-15$ for $\mathrm{HI}$ animals from 6 to $72 \mathrm{~h}$ ). (b) Correlation of the serum levels of 24S-HC with the expression of SBDP $145 / 150 \mathrm{kD}$ at $6 \mathrm{~h}$ (top), at $24 \mathrm{~h}$ (middle), and with the expression of SBDP $120 \mathrm{kD}$ at $24 \mathrm{~h}$ (bottom). Sample number, $R^{2}$, and $P$ values are shown in the graphs. 24S-HC, 24Shydroxycholesterol; $\mathrm{HI}$, hypoxia-ischemia; OD, optical density; SBDP, spectrin breakdown product.

$n=11$ ), and with cleaved caspase- 3 at the same time points (Figure $6 \mathrm{~b}$, at $6 \mathrm{~h}: R^{2}=0.738, P=0.002, n=10$; at $24 \mathrm{~h}$ : $\left.R^{2}=0.462, P=0.044, n=9\right)$. The correlation between serum levels of $24 \mathrm{~S}-\mathrm{HC}$ and SBDP $120 \mathrm{kD}$ was also significant at $24 \mathrm{~h}$ (Figure 5b, $R^{2}=0.557, P=0.008, n=11$ ).

\section{DISCUSSION}

We demonstrated an enhanced brain cholesterol turnover following neonatal $\mathrm{HI}$ as evidenced by the upregulation of CYP46A1 in the ipsilateral cortex, leading to an increased production of $24 \mathrm{~S}-\mathrm{HC}$ in the injured hemisphere and simultaneous excretion into the bloodstream. The correction between the levels of serum 24S-HC and brain necrosis/ apoptosis at early post-HI stage supports a possible value of plasma level of $24 \mathrm{~S}-\mathrm{HC}$ as a novel and early biomarker for severity of neonatal $\mathrm{HI}$ brain damage.

Present mainly in the brain, CYP46A1 catalyzes the hydroxylation of cholesterol into $24 \mathrm{~S}-\mathrm{HC}$ to eliminate surplus cholesterol from the brain, and thereby has an important role in maintenance of cholesterol homeostasis $(19,20)$. CYP46A1 protein expression is low before 1 week after birth in mice and gradually increases to reach a steady level around 3 weeks $(6,8)$. In humans, it reaches the adult level at around 3 years of age (8). This allows deposition of nearly all cholesterol at an early age to assist in the rapid expansion of the brain and myelin, and in the removal of redundant cholesterol as the animal matures. We showed an upregulation of CYP46A1 following neonatal $\mathrm{HI}$ at $\mathrm{P} 9$ sustaining at least 24 or $48 \mathrm{~h}$. It is unknown how this enzyme is activated by HI, whether this response is simply adaptative, or is it involved in processes of brain injury or protection. CYP46A1 promoter activity appears to be resistant to changes of molecules implicated in regulation of cholesterol homeostasis including cholesterol and 24S-HC (6), but can be induced by the Sp-family of transcriptional factors (21) and epigenetic modifications (22). Recent studies have suggested two mechanisms for CYP46A1 


\section{Cholesterol metabolism in neonatal brain}

Articles

a
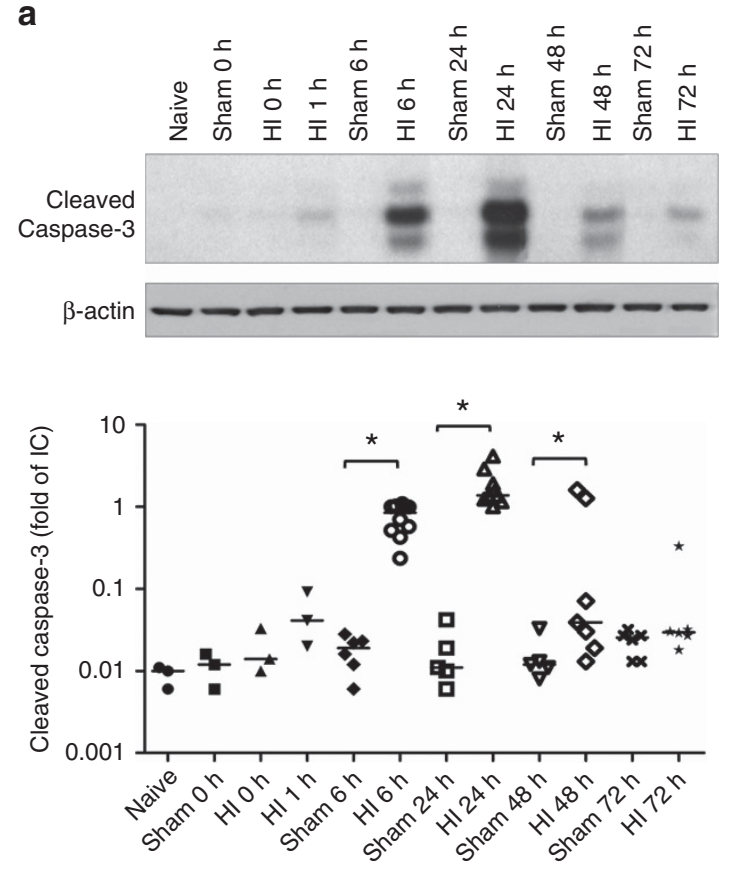

b
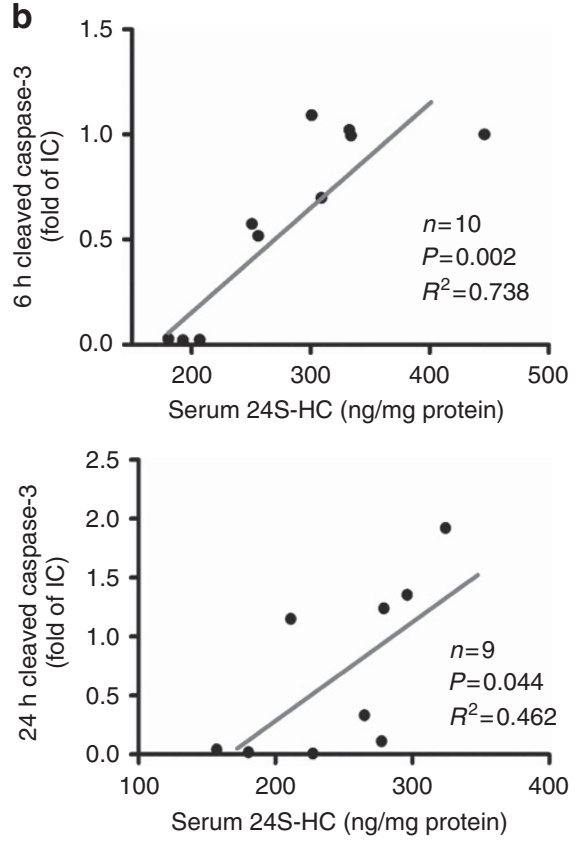

Figure 6. Correlation of the serum levels of $24 \mathrm{~S}-\mathrm{HC}$ with the expression of cleaved caspase-3 at 6 and $24 \mathrm{~h}$ after neonatal $\mathrm{HI}$. (a) Protein expression of cleaved caspase- 3 in the cortices was measured by western blotting at the indicated time points and presented as the OD ratio to $\beta$-actin and normalized to an IC. Sham vs. HI, ${ }^{*} P=0.0112$ at $6 \mathrm{~h},{ }^{*} P=0.0027$ at $24 \mathrm{~h},{ }^{*} P=0.0228$ at $72 \mathrm{~h}$ ( $n=5-6$ for sham animals, $n=7-10$ for HI animals from 6 to $72 \mathrm{~h}$ ). (b) Correlation of the serum levels of $24 \mathrm{~S}-\mathrm{HC}$ with the expression of cleaved caspase-3 at $6 \mathrm{~h}$ (top) and at $24 \mathrm{~h}$ (bottom). Sample number, $R^{2}$, and $P$ values are shown in the graphs. 24S-HC, 24S-hydroxycholesterol; HI, hypoxia-ischemia; IC, internal control; OD, optical density.

activation: oxidative stress (6) and glutamate (15), both of which are associated with cell death in brain ischemia, and with overactivation of the NMDA receptors, the major ionotropic glutamate receptors mediating excitotoxicity in HI brain injury. CYP46A1 enzymatic product $24 \mathrm{~S}-\mathrm{HC}$ was found to be a potent allosteric modulator to enhance the function of NMDA receptors $(23,24)$. These findings suggest a reciprocal activation of the NMDA receptor pathway and the cholesterol metabolism pathway, which may form a viscous cycle to exacerbate brain damage following neonatal HI.

Cholesterol loss is expected to be the direct consequence of CYP46A1 activation. This might be partially responsible for the reduction in cholesterol in the ipsilateral cortex at $6 \mathrm{~h}$ after HI. The changes of the total cholesterol amount found here reflected the balance between de novo synthesis and breakdown by CYP46A1. We did not measure cholesterol that was newly synthesized after neonatal HI; however, elevated generation of $24 \mathrm{~S}-\mathrm{HC}$ could downregulate cholesterol synthesis via blocking the proteolytic activation of transcription factor sterol regulatory element-binding protein 2 (SREBP2), which controls the pathway of cholesterol biosynthesis (25). In connection with excitotoxicity, a $\mathrm{Ca}^{2+}$-dependent cholesterol loss was found in cultured hippocampal neurons exposed to NMDA, which was paralleled by translocation of CYP46A1 from the endoplasmic reticulum, where it normally resides, to the plasma membrane and release of $24 \mathrm{~S}-\mathrm{HC}$ into the medium (26). Apparently, we cannot draw the conclusion that cholesterol loss at $6 \mathrm{~h}$ was solely attributable to the action of CYP46A1. The level of HMGCR was unchanged at $6 \mathrm{~h}$, but increased at $24 \mathrm{~h}$ after $\mathrm{HI}$, which might prevent further reduction of cholesterol in the injured hemisphere. The other two studies of neonatal HI in P7 Sprague Dawley (SD) rats also reported the diminished cholesterol contents, which lasted longer for 3 days or 3 months following the initial insult $(13,14)$. Disturbance of cholesterol homeostasis after neonatal HI may have adverse consequences on cell survival and brain development. For example, accelerated cholesterol hydroxylation found in this study produced a high level of 24S-HC, which has been shown to be cytotoxic to neurons by inducing apoptosis, necroptosis (a form of programmed necrosis) $(27,28)$, inflammatory responses (29), and cognitive impairment (30). The colocalization of increased CYP46A1 with cleaved caspase-3 in the ipsilateral cortex indicates that CYP46A1 may be associated with cell death following HI. Further studies are required to elucidate the functional roles of CYP46A1 and 24S-HC in neonatal brain HI, especially in long-term neurological deficits.

Although primarily confined to neurons in the healthy brain, CYP46A1 has been found in other cell types in different disease conditions, for example, in activated microglia and reactive astrocytes in brain trauma $(17,18)$, in astrocytes of Alzheimer's disease patients (31), or in macrophages/microglia in an animal model of multiple sclerosis (32). They were proposed to clear extracellular cholesterol released from the damaged cell membranes, which has not been proved experimentally. We show that CYP46A1 is localized in 
neurons and oligodendrocytes, but not in astrocytes and microglia. White matter is a common lesion site in rodent neonatal HI (33), and in human infants, especially preterm babies (34), who suffered from HIE. As oligodendrocytes are sensitive to oxidative stress and excitotoxicity in the developing brain (35), if CYP46A1 is upregulated in oligodendrocytes after $\mathrm{HI}$, it may break down cholesterol leading to myelin degradation or limiting its availability for myelin synthesis and white matter development as cholesterol in oligodendrocytes is a rate-limiting factor for brain maturation (2). Targeted inhibition or deletion of CYP46A1 in oligodendrocyte may reveal its precise function in the normal brain and under disease conditions. Different cell populations play distinct roles in regulating cholesterol homeostasis. For example, neurons can utilize external cholesterol transported from glial cells $(36,37)$. Similarly, brain cells may coordinate for cholesterol clearance because not all brain cells, even not all neurons, express CYP46A1 $(8,20)$.

Studies with CYP46A1 knockout mice have suggested that this enzyme is responsible for the production of $\approx 98-99 \%$ of brain $24 \mathrm{~S}-\mathrm{HC}$ and $\approx 60-80 \% 24 \mathrm{~S}-\mathrm{HC}$ in the serum $(9,38)$. Our data support the cerebral origin of blood $24 \mathrm{~S}-\mathrm{HC}$ owing to the concomitant increase and correlation of $24 \mathrm{~S}-\mathrm{HC}$ in the serum and in the brain. Although it is unknown whether an acute elevation of serum $24 \mathrm{~S}-\mathrm{HC}$ can predict histological and functional outcomes at later time points, based on its brain specificity, and a strong correlation with necrotic and apoptotic cell death early after HI, this pilot work suggests that blood 24S-HC might be a biologically plausible marker for HIE to assist clinical diagnosis. SBDP can be detected in the serum of human neonates and the amounts are associated with the severity of HIE clinical syndromes with good sensitivity and specificity (39). However, serum SBDP can be derived from extracerebral organs when damaged. Radiological or functional measures are required to explore the feasibility of using blood $24 \mathrm{~S}-\mathrm{HC}$ as a diagnostic tool in HIE patients. Targeted lipid profiling discovered plasma sphingomyelins as potential markers for acute stroke patients (40). Lipids could be better tools for diagnosis than protein markers because of their abundance in the brain, small size for readily crossing the blood-brain barrier, and higher sensitivity to oxidative stress for a possible earlier detection.

Taken together, this is the first study investigating a brainspecific cholesterol turnover pathway following neonatal $\mathrm{HI}$. Activation of CYP46A1 may have more specialized roles than just removing cholesterol from the central nervous system. Future work is required to gain better insight into cholesterol homeostasis in the developing brain, which is subjected to a more sophisticated regulation than that in the adult brain where cholesterol is largely metabolically inert.

\section{STATEMENT OF FINANCIAL SUPPORT}

This work was supported by the National Institute of Neurological Disorders and Stroke (RO1NS084057 to Dr. Jiang).
Disclosure: The authors declare no competing financial interests.

\section{REFERENCES}

1. Mauch DH, Nagler K, Schumacher S, et al. CNS synaptogenesis promoted by glia-derived cholesterol. Science 2001;294:1354-7.

2. Saher G, Brugger B, Lappe-Siefke C, et al. High cholesterol level is essential for myelin membrane growth. Nat Neurosci 2005;8:468-75.

3. Dietschy JM. Central nervous system: cholesterol turnover, brain development and neurodegeneration. Biol Chem 2009;390:287-93.

4. Dietschy JM, Turley SD. Thematic review series: brain lipids. Cholesterol metabolism in the central nervous system during early development and in the mature animal. J Lipid Res 2004;45:1375-97.

5. Quan G, Xie C, Dietschy JM, Turley SD. Ontogenesis and regulation of cholesterol metabolism in the central nervous system of the mouse. Brain Res Dev Brain Res 2003;146:87-98.

6. Ohyama Y, Meaney S, Heverin M, et al. Studies on the transcriptional regulation of cholesterol 24-hydroxylase (CYP46A1): marked insensitivity toward different regulatory axes. J Biol Chem 2006;281:3810-20.

7. Jurevics H, Morell P. Cholesterol for synthesis of myelin is made locally, not imported into brain. J Neurochem 1995;64:895-901.

8. Lund EG, Guileyardo JM, Russell DW. cDNA cloning of cholesterol 24hydroxylase, a mediator of cholesterol homeostasis in the brain. Proc Natl Acad Sci U S A 1999;96:7238-43.

9. Bjorkhem I, Lutjohann D, Diczfalusy U, Stahle L, Ahlborg G, Wahren J. Cholesterol homeostasis in human brain: turnover of 24 S-hydroxycholesterol and evidence for a cerebral origin of most of this oxysterol in the circulation. J Lipid Res 1998;39:1594-600.

10. Lutjohann D, von Bergmann K. 24S-hydroxycholesterol: a marker of brain cholesterol metabolism. Pharmacopsychiatry 2003;36 (Suppl 2): S102-6.

11. Leoni V, Caccia C. 24S-hydroxycholesterol in plasma: a marker of cholesterol turnover in neurodegenerative diseases. Biochimie 2013;95: 595-612.

12. DeBose-Boyd RA. Feedback regulation of cholesterol synthesis: sterolaccelerated ubiquitination and degradation of HMG CoA reductase. Cell Res 2008;18:609-21.

13. Ramirez MR, Muraro F, Zylbersztejn DS, et al. Neonatal hypoxiaischemia reduces ganglioside, phospholipid and cholesterol contents in the rat hippocampus. Neurosci Res 2003;46:339-47.

14. Yu Z, Li S, Lv SH, et al. Hypoxia-ischemia brain damage disrupts brain cholesterol homeostasis in neonatal rats. Neuropediatrics 2009;40:179-85.

15. Mast N, Anderson KW, Johnson KM, Phan TTN, Guengerich FP, Pikuleva IA. In vitro cytochrome P450 46A1 (CYP46A1) activation by neuroactive compounds. J Biol Chem 2017;292:12934-46.

16. Millar LJ, Shi L, Hoerder-Suabedissen A, Molnar Z. Neonatal hypoxia ischaemia: mechanisms, models, and therapeutic challenges. Front Cell Neurosci 2017;11:78.

17. Cartagena CM, Ahmed F, Burns MP, et al. Cortical injury increases cholesterol $24 \mathrm{~S}$ hydroxylase (Cyp46) levels in the rat brain. J Neurotrauma 2008;25:1087-98.

18. Smiljanic K, Lavrnja I, Mladenovic Djordjevic A, et al. Brain injury induces cholesterol 24-hydroxylase (Cyp46) expression in glial cells in a time-dependent manner. Histochem Cell Biol 2010;134:159-69.

19. Moutinho M, Nunes MJ, Rodrigues E. Cholesterol 24-hydroxylase: brain cholesterol metabolism and beyond. Biochim Biophys Acta 2016;1861: 1911-20.

20. Russell DW, Halford RW, Ramirez DM, Shah R, Kotti T. Cholesterol 24hydroxylase: an enzyme of cholesterol turnover in the brain. Annu Rev Biochem 2009;78:1017-40.

21. Milagre I, Nunes MJ, Gama MJ, et al. Transcriptional regulation of the human CYP46A1 brain-specific expression by Sp transcription factors. J Neurochem 2008;106:835-49.

22. Shafaati M, O'Driscoll R, Bjorkhem I, Meaney S. Transcriptional regulation of cholesterol 24-hydroxylase by histone deacetylase inhibitors. Biochem Biophys Res Commun 2009;378:689-94. 
23. Linsenbardt AJ, Taylor A, Emnett CM, et al. Different oxysterols have opposing actions at $\mathrm{N}$-methyl-D-aspartate receptors. Neuropharmacology 2014;85:232-42.

24. Paul SM, Doherty JJ, Robichaud AJ, et al. The major brain cholesterol metabolite 24(S)-hydroxycholesterol is a potent allosteric modulator of N-methyl-D-aspartate receptors. J Neurosci 2013;33:17290-300.

25. Radhakrishnan A, Ikeda Y, Kwon HJ, Brown MS, Goldstein JL. Sterol-regulated transport of SREBPs from endoplasmic reticulum to Golgi: oxysterols block transport by binding to Insig. Proc Natl Acad Sci USA 2007;104:6511-8.

26. Sodero AO, Vriens J, Ghosh D, et al. Cholesterol loss during glutamatemediated excitotoxicity. EMBO J 2012;31:1764-73.

27. Noguchi N, Urano Y, Takabe W, Saito Y. New aspects of 24(S)hydroxycholesterol in modulating neuronal cell death. Free Radic Biol Med 2015;87:366-72.

28. Yamanaka K, Saito Y, Yamamori T, Urano Y, Noguchi N. 24(S)hydroxycholesterol induces neuronal cell death through necroptosis, a form of programmed necrosis. J Biol Chem 2011;286:24666-73.

29. Alexandrov P, Cui JG, Zhao Y, Lukiw WJ. 24S-hydroxycholesterol induces inflammatory gene expression in primary human neural cells. Neuroreport 2005;16:909-13.

30. Zhao S, Liao W, Xu N, et al. Polar metabolite of cholesterol induces rat cognitive dysfunctions. Neuroscience 2009;164:398-403.

31. Brown J 3rd, Theisler C, Silberman S, et al. Differential expression of cholesterol hydroxylases in Alzheimer's disease. J Biol Chem 2004;279: 34674-81.
32. Lavrnja I, Smiljanic K, Savic D, et al. Expression profiles of cholesterol metabolism-related genes are altered during development of experimental autoimmune encephalomyelitis in the rat spinal cord. Sci Rep 2017;7: 2702.

33. Liu Y, Silverstein FS, Skoff R, Barks JD. Hypoxic-ischemic oligodendroglial injury in neonatal rat brain. Pediatr Res 2002;51:25-33.

34. Back SA. White matter injury in the preterm infant: pathology and mechanisms. Acta Neuropathol 2017;134:331-49.

35. Silbereis JC, Huang EJ, Back SA, Rowitch DH. Towards improved animal models of neonatal white matter injury associated with cerebral palsy. Dis Model Mech 2010;3:678-88.

36. Funfschilling U, Jockusch WJ, Sivakumar N, et al. Critical time window of neuronal cholesterol synthesis during neurite outgrowth. J Neurosci 2012;32:7632-45.

37. Pfrieger FW, Ungerer N. Cholesterol metabolism in neurons and astrocytes. Prog Lipid Res 2011;50:357-71.

38. Lund EG, Xie C, Kotti T, Turley SD, Dietschy JM, Russell DW. Knockout of the cholesterol 24-hydroxylase gene in mice reveals a brainspecific mechanism of cholesterol turnover. J Biol Chem 2003;278: 22980-8.

39. Wu H, Li Z, Yang X, Liu J, Wang W, G Liu. SBDPs and Tau proteins for diagnosis and hypothermia therapy in neonatal hypoxic ischemic encephalopathy. Exp Ther Med 2017;13:225-9.

40. Sheth SA, Iavarone AT, Liebeskind DS, Won SJ, Swanson RA. Targeted lipid profiling discovers plasma biomarkers of acute brain injury. PLoS ONE 2015;10:e0129735. 